\title{
Aplicabilidad de la energía cinética en el inicio de la inestabilidad de materiales granulares en un tambor rotador mediante la técnica PIV (Particle Image Velocimetry)
}

\section{The applicability of kinetic energy for granular materials at the beginning of the instability process in a rotating drum by means of Particle Image Velocimetry (PIV)}

\author{
Camilo Correa Uribe ${ }^{[1]}$, Mateo Maldonado Rodríguez ${ }^{[2]}$, Luis Felipe Prada Sarmiento ${ }^{[3]}$ \\ y Alfonso Mariano Ramos Cañón ${ }^{[4]}$
}

\begin{abstract}
Resumen
Se presenta un estudio del comportamiento de materiales granulares sometidos a procesos de inestabilidad en un tambor rotador. Mediante un procedimiento novedoso se evalúa la aplicabilidad de la energía cinética como parámetro de medición de procesos de inestabilidad y su relación con las granulometrías de los materiales y las velocidades de rotación del tambor. Determinadas granulometrías se ensayan en diferentes montajes, para la captura de imágenes consecutivas del proceso de flujo de los materiales presentes en el tambor rotador. El procesamiento de las imágenes es realizado mediante la técnica de Particle Image Velocimetry (PIV) para así poder determinar los vectores de desplazamiento y el campo de velocidad de las partículas, utilizando el toolbox de Matlab llamado Pivlab. Posteriormente se calcula la energía cinética y se determina su aplicabilidad mediante el análisis de la variación de este parámetro en los procesos de inestabilidad y su relación con las granulometrías de los materiales y las velocidades de rotación del tambor. En los ensayos realizados se determinaron dos tipos de flujo de partículas: régimen de caída y régimen rodante. Además, se encontró la dependencia del tipo de material en el valor umbral de energía cinética al inicio del proceso de inestabilidad y la independencia de este parámetro del ángulo de inclinación del material dentro del tambor rotador. Los resultados de este proyecto permiten un análisis de los procesos de inestabilidad en materiales granulares mediante metodologías no convencionales.
\end{abstract}

Palabras clave: Tambor rotador; Flujo granular; Velocidad; Energía cinética; PIV.

[1] Estudiante de Ingeniería Civil. Pontificia Universidad Javeriana. camilo.correa@javeriana.edu.co

[2] Estudiante de Ingeniería Civil. Pontificia Universidad Javeriana. maldonado.m@javeriana.edu.co

[3] Profesor asistente. Departamento de Ingeniería Civil. Pontificia Universidad Javeriana. lf.pradas@javeriana.edu.co

[4] Profesor asociado. Instituto Geofísico. Pontificia Universidad Javeriana. a-ramos@javeriana.edu.co

Recibido: 21 enero 2015 - Aceptado: 5 febrero 2015 


\begin{abstract}
This article presents a study of the behavior of granular materials that are subject to a process of instability in a rotating drum. By employing an original procedure, the applicability of kinetic energy as a parameter to measure processes of instability and their relationships with the granulometry of materials and rotating drum's velocities are evaluated. Certain granulometries are tested in various set-ups in order to capture consecutive images of the process in which the present materials flow into the rotating drum. Image processing is carried out by using the Particle Image Velocimetry (PIV) technique in order to be able to determine the particles' displacement vectors and velocity fields by using Pivlab, the Matlab toolbox. Subsequently, the kinetic energy is calculated and its applicability is determined through analyzing the variation of this parameter in the processes of instability and its relationship with the granulometry of materials and the velocities of the rotating drum. The trials undertaken as part of this study have determined that there are two types of particle flow: falling and rolling. Moreover, the dependence of the material type on the threshold value of kinetic energy at the beginning of the process of instability was discovered as well as the independence of this parameter from the angle of inclination of the material inside the rotating drum. The results from this research allow for an analysis of the processes of instability in granular materials by means of non-conventional methodologies.
\end{abstract}

Key words: Rotating Drum; Granular flow; Velocity; Kinetic energy; PIV.

\section{Introducción}

Los fenómenos de deslizamiento y flujo de detritos están ubicados como el sexto evento natural más ocurrente y con mayores pérdidas humanas registradas a nivel mundial (Centre for reearch for epidemiology of disasters, 2013). Estos conllevan igualmente a la destrucción de territorios enteros resultado de la inestabilidad existente en taludes con altas pendientes y a la variación de las condiciones hidrogeomorfológicas de éstos (Jakob \& Hungr, 2005).

El estudio del comportamiento mecánico de estos fenómenos se ha profundizado especialmente en países como Estados Unidos e Inglaterra (Jakob \& Hungr, 2005). Los estudios de inestabilidad en materiales granulares se realizan principalmente mediante análisis numéricos bajo herramientas computacionales que abarcan desde los Sistemas de Información Geográfica (SIG) (Bateman, 2007) hasta Métodos de Elementos Finitos y Métodos de Elementos Discretos (MEF \& MED, respectivamente) (Leonir, et al., 2011)

En la modelación MED de procesos de inestabilidad de materiales granulares se puede hacer un seguimiento detallado de algunas variables de estado relevantes como la velocidad a partir de la cual se puede generar un potencial de energía cinética, que puede indicar un punto de inflexión diferenciador de zonas estables e inestables del talud. Para esto, se han utilizados diferentes técnicas experimentales que incluyen desde montajes experimentales en canales de pendiente variable, hasta el uso de tambores rotadores (Xu, et al., 2011).

Una vez simulado el flujo de detritos mediante el tambor, se hace necesaria la medición de los desplazamientos de los materiales a escala granular a partir de técnicas de medición ópticas utilizadas en el campo de la geotecnia como Particle Image Velocimetry (PIV) (White, et al., 2003) y específicamente en la deformación de dichos materiales granulares (Slominski, et al., 2006).

\subsection{Técnica de estudio de flujo con PIV}

El PIV es una técnica de medición indirecta de las velocidades de flujo, ya que el método permite determinar la velocidad de las partículas existentes dentro del flujo mediante la medición de variables de distancia y tiempo.

El método de PIV se basa en el análisis de imágenes tomadas consecutivamente con una diferencia de tiempo $\Delta t$, mediante patrones de reconocimiento ópticos que permiten la identificación del desplazamiento de las partículas (Raffel, et al., 1998). Estos patrones, llamados campos de intensidad, son asignados en áreas de interrogación definidas según el tamaño de los materiales.

El análisis realizado en Pivlab se basa en la correlación directa de imágenes, la cual provee resultados 
con mayor precisión en la magnitud y dirección de los vectores de desplazamientos (Pust, 2000). A su vez, las imágenes se pueden reducir a un dominio infinito de análisis mediante la descomposición de los campos de intensidad del dominio del tiempo al dominio de la frecuencia, utilizando la transformada rápida de Fourier.

\section{Materiales y métodos}

\subsection{Materiales y equipos}

El tambor rotador es un cilindro de acero de $60 \mathrm{~cm}$ de diámetro y $10 \mathrm{~cm}$ de profundidad con la cara exterior de vidrio templado de alta resistencia. El tambor rota de forma continua por medio de un motor controlado con un potenciómetro que regula la velocidad de rotación angular independientemente del peso del material depositado en el cilindro. Las velocidades de rotación del tambor están reguladas por 7 niveles de una perilla de control, tal como se presenta en la figura 1. Dichas velocidades se clasificaron según su magnitud en tres niveles: baja, alta y elevada.

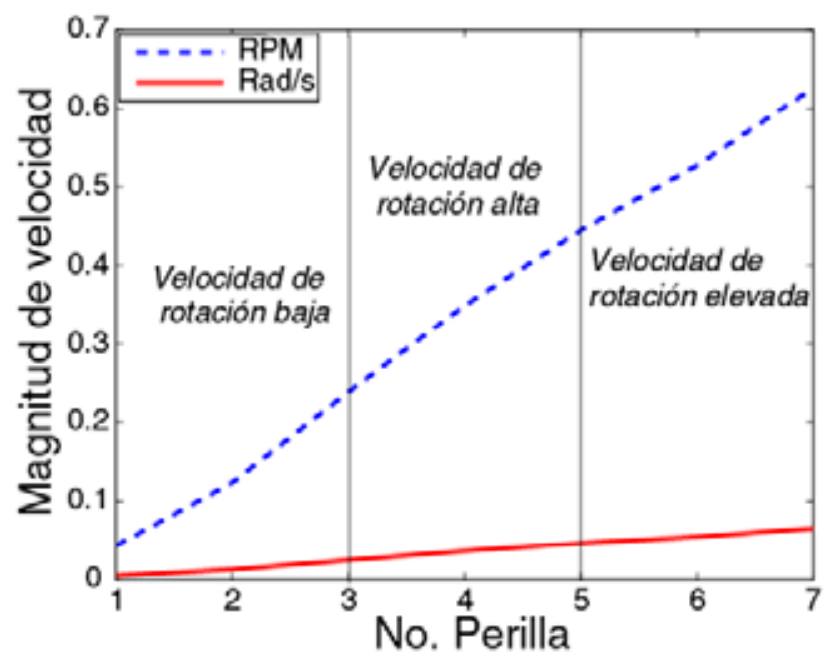

Figura 1. Velocidades de rotación del tambor.

Para propósitos de montaje dentro del tambor rotador y su procesamiento mediante PIV se escogieron tres materiales granulares diferentes: esferas de plástico, canicas y dos granulometrías de la arena del Guamo (ver figura 2), una original con $C_{u}=2.8, C_{C}=1.2 \mathrm{y}$ otra versión modificada con $C_{U}=3.68, C_{C}=0.5$.

Estos materiales fueron seleccionados con el fin de poder tener un control en cuanto a sus características físicas y propiedades geomecánicas.

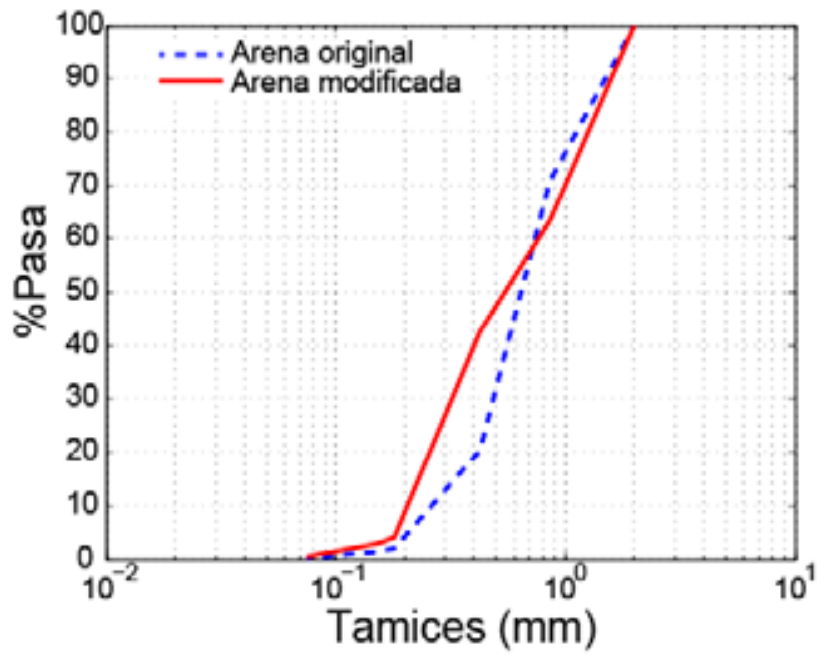

Figura 2. Granulometrías empleadas de la arena del Guamo.

\subsection{Montaje del ensayo}

Dentro del tambor rotador se hacen las primeras pruebas y mediciones usando esferas plásticas y canicas con un porcentaje de llenado menor a la mitad del volumen del tambor, cuya configuración se detalla en la tabla 1 para materiales uniformes y en la tabla 2 para mezclas de tamaños de canicas.

Tabla 1. Distribución uniforme de materiales en el tambor rotador.

\begin{tabular}{lcc}
\hline Material & \% Material & \% Volumen \\
\hline Esferas plásticas & 100 & 27.5 \\
Canicas grandes & 100 & 38.9 \\
Canicas pequeñas & 100 & 41.0 \\
\hline
\end{tabular}

Tabla 2. Distribución de mezcla de materiales en el tambor rotador.

\begin{tabular}{lccc}
\hline $\begin{array}{l}\text { Mezcla } \\
\text { Material }\end{array}$ & Material & $\begin{array}{c}\text { Porcentaje } \\
\text { Material }\end{array}$ & $\begin{array}{c}\text { Porcentaje } \\
\text { Volumen }\end{array}$ \\
\hline Mezcla A & $\begin{array}{c}\text { Canicas grandes } \\
\text { Canicas pequeñas }\end{array}$ & 60 & 40.0 \\
& 40 & \\
Mezcla B & Canicas grandes & 40 & 40.4 \\
& Canicas pequeñas & 60 & \\
\hline
\end{tabular}

Una vez depositado el material dentro del tambor, se activa el motor de rotación para generar avalanchas de forma continua. Estos ciclos de avalanchas se graban 
en video empleando una cámara de alta velocidad Motionblitz Cube No.4 (permite registrar hasta 10000 imágenes por segundo) y un equipo de luces día Dedolight de emisión directa de alta potencia (600W).

La iluminación debe ubicarse adecuadamente para no generar reflexión en la superficie del vidrio templado del tambor. Se debe garantizar además que el área de interés para el análisis con PIV sea lo suficiente iluminada de forma tal que no se presenten zonas oscuras. El montaje del tambor rotador, la cámara de alta velocidad y el sistema de luces se presenta en la figura 3.

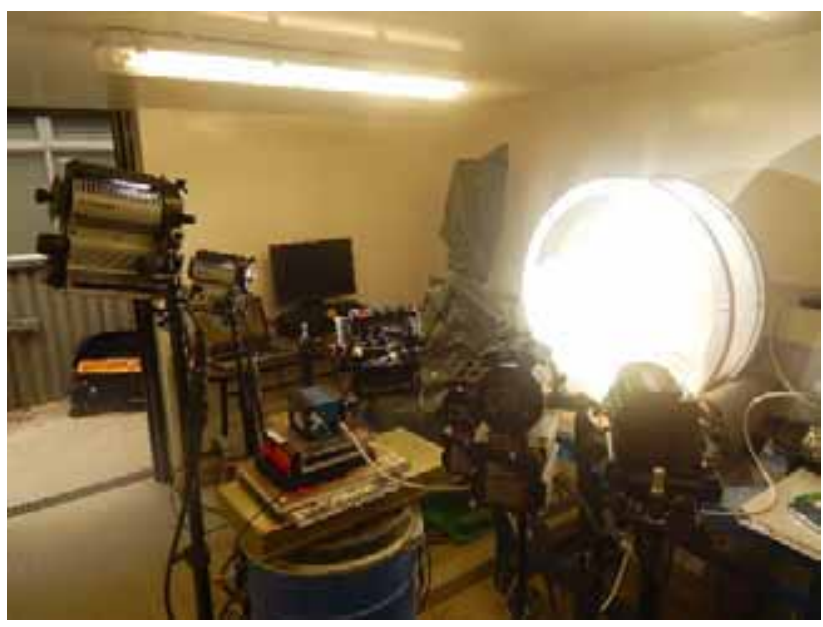

Figura 3. Montaje de ensayo para la grabación de imágenes de alta velocidad.

\subsection{Procesamiento de imágenes}

Las imágenes son procesadas por medio del toolbox Pivlab del programa Matlab para poder obtener los vectores de desplazamiento (ver figura 4) y el campo de velocidades (ver figura 5) de las partículas granulares en diferentes instantes de tiempo.

Mediante la ayuda del software Matlab a donde se exportan los resultados del PIV, se realiza una programación de los cálculos necesarios para obtener una medida de la energía cinética por unidad de masa $\left(E_{C} / m\right)$ de los materiales granulares.

Por definición,

$$
E_{C}=m \cdot V^{2}
$$

donde $m$ es la masa del material granular y $v$ es la velocidad de traslación de las partículas.

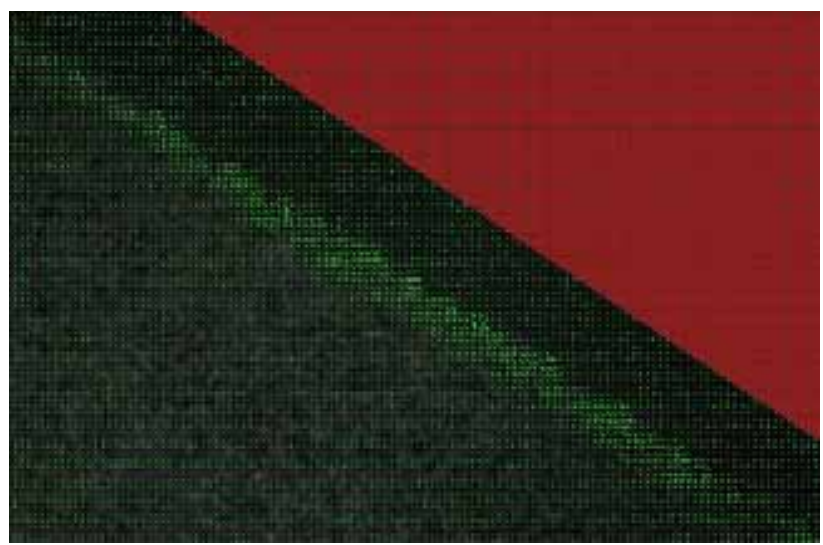

Figura 4. Vectores de desplazamiento en PIV. Análisis en arenas.

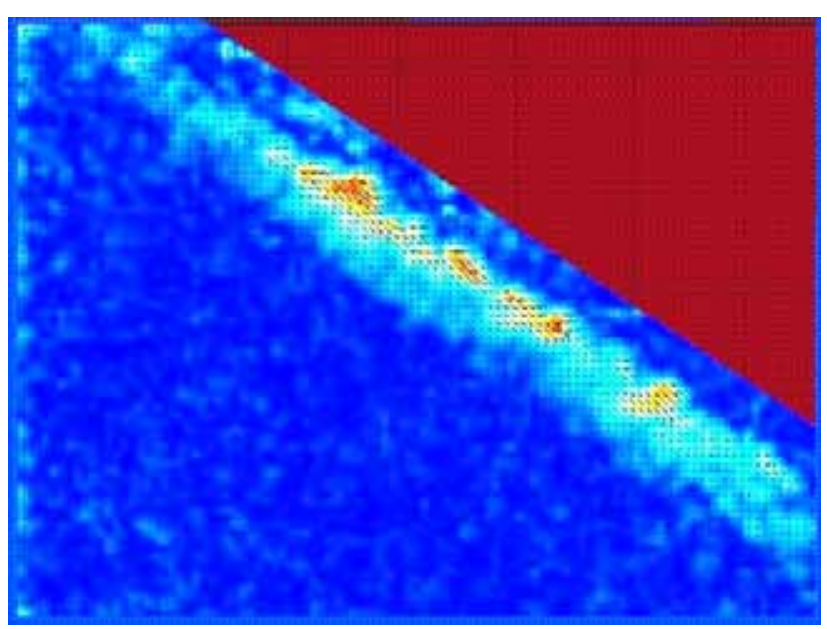

Figura 5. Campo de velocidad en PIV. Análisis en arenas.

$\mathrm{Al}$ normalizar la energía cinética entre la masa del material granular se llega a:

$\frac{E_{C}=V^{2}}{\mathrm{~m} 2}$

En donde la velocidad traslacional se puede descomponer vectorialmente en sus componentes horizontales y verticales:

$V^{2}=V_{x}^{2}+V_{y}^{2}$

Para cada área de análisis del PIV se calcula la energía cinética correspondiente y se grafican los resultados en función del tiempo (“área”). De igual modo, se suman los resultados de aplicar las ecuaciones anteriores para cada área de análisis y se obtiene un valor total por cuadro ("frame") en función del tiempo, tal como se observa en la figura 6 para el análisis de imágenes de deslizamientos producidos con esferas de plástico. 


\section{Resultados y Discusión}

Para analizar el proceso completo de inestabilidad de los materiales se calculan las gráficas de evolución de energía cinética por "frame" en función del tiempo (ver figura 6), en la cual se pueden diferenciar los diferentes momentos de generación, apogeo y decaimiento del proceso de avalanchas.

Del análisis de la evolución de la energía cinética durante los procesos de avalancha se identificaron cuatro etapas.

Inicialmente el material se encuentra en estado de reposo y la rotación del tambor genera ruido en la energía cinética de las partículas (etapa A). Posteriormente existe un instante de tiempo en el que la energía cinética comienza a tener una variación alta para instantes de tiempo consecutivos y finalmente un punto teórico a partir del cual se supera un umbral de energía drásticamente (etapa B). Seguido a esto existe un tiempo más largo en el que la energía cinética aumenta significativamente hasta alcanzar valores de energía cinética máximos y desciende gradualmente hasta buscar la estabilidad (etapa C). Finalmente el material tien- de a un estado de reposo para comenzar un nuevo proceso de inestabilidad (etapa D).

La energía cinética registrada durante los instantes de tiempo en el proceso de inestabilidad, se puede asociar con los ángulos de la superficie del material granular dentro del tambor rotador en dichos instantes mediante el esquema de la figura 7.

En la fase A del proceso de inestabilidad, el ángulo de material (línea roja) se encuentra en un estado de reposo. Posteriormente en la etapa $\mathrm{B}$, el ángulo tiende a incrementar pocos grados de inclinación para alcanzar un ángulo pico de la superficie del material (línea azul). En el instante donde se inicia el flujo desagregado de las partículas en la etapa $C$, el ángulo de inclinación fluctúa entre la condición inicial de reposo y el ángulo máximo (línea verde). Finalmente en el tiempo D, el material tiende a estabilizarse y a encontrar su ángulo de inclinación inicial.

Es posible en todo el proceso determinar características de flujo como la profundidad en milímetros en función del diámetro del material ensayado. Esta profundidad de flujo cubre desde la superficie libre

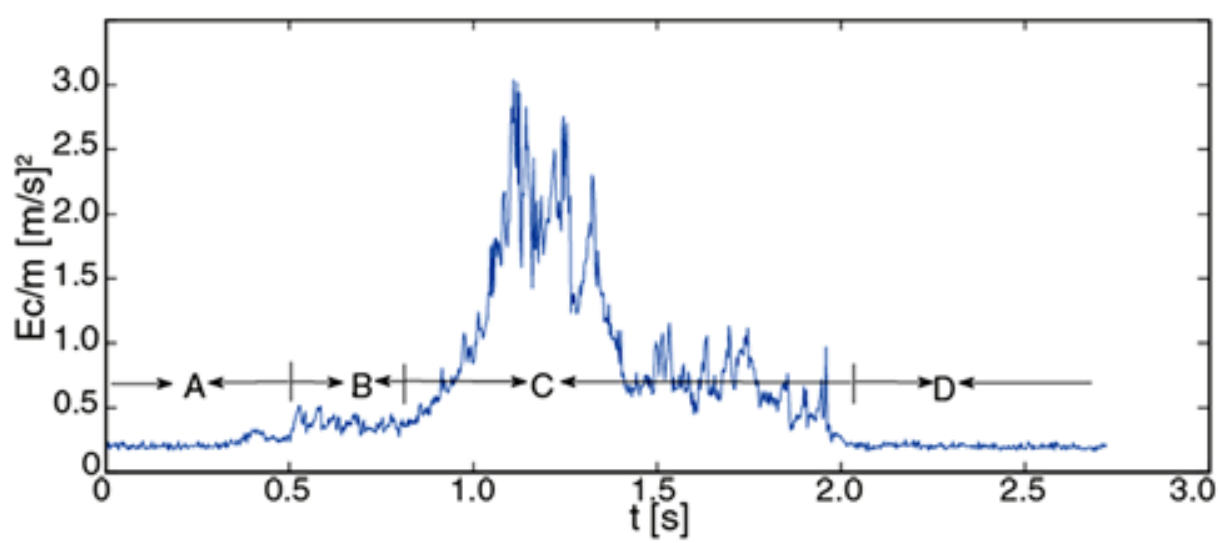

Figura 6. Evolución temporal de la energía cinética normalizada entre la masa durante el proceso de inestabilidad en materiales granulares.

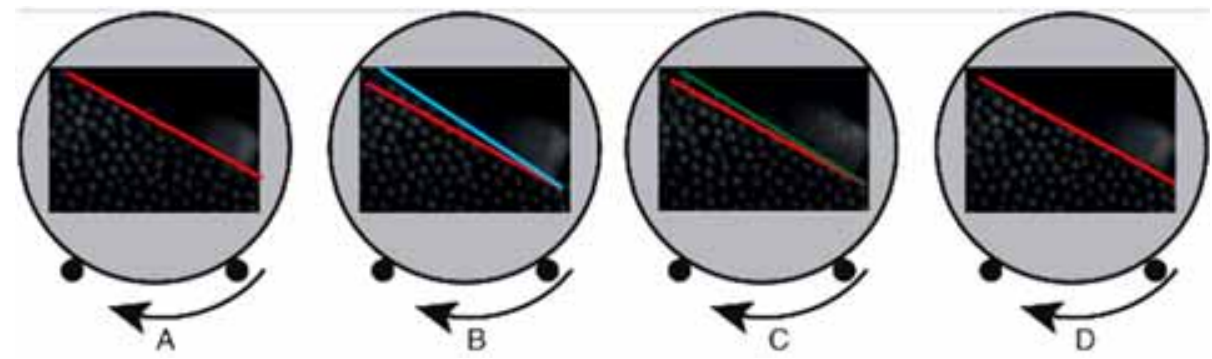

Figura 7. Relación de ángulos de inclinación de la superficie del material granular dentro del proceso de inestabilidad. 
hasta la cuña de falla determinada por el decremento de energía que existe en el flujo de partículas, tal como se observa en la figura 8.

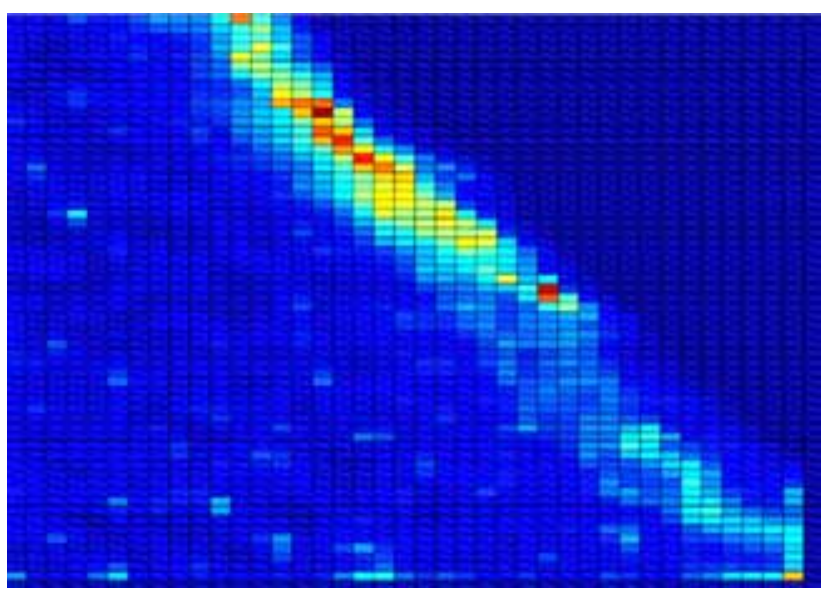

Figura 8. Superficie de flujo de energía cinética total por imagen. Análisis en canicas.

\subsection{Velocidad de rotación}

Con el fin de evaluar los efectos de la velocidad de rotación del tambor sobre el perfil promedio de la energía cinética de un evento de inestabilidad, se hicieron varias mediciones en el tambor empleando las canicas de tamaño pequeño, grande y mezclas de tamaños bajo diferentes niveles de velocidad de rotación del tambor.

En el caso de las observaciones de las avalanchas generadas con las canicas pequeñas se desarrollan velocidades de rotación baja, alta y elevada (ver figura

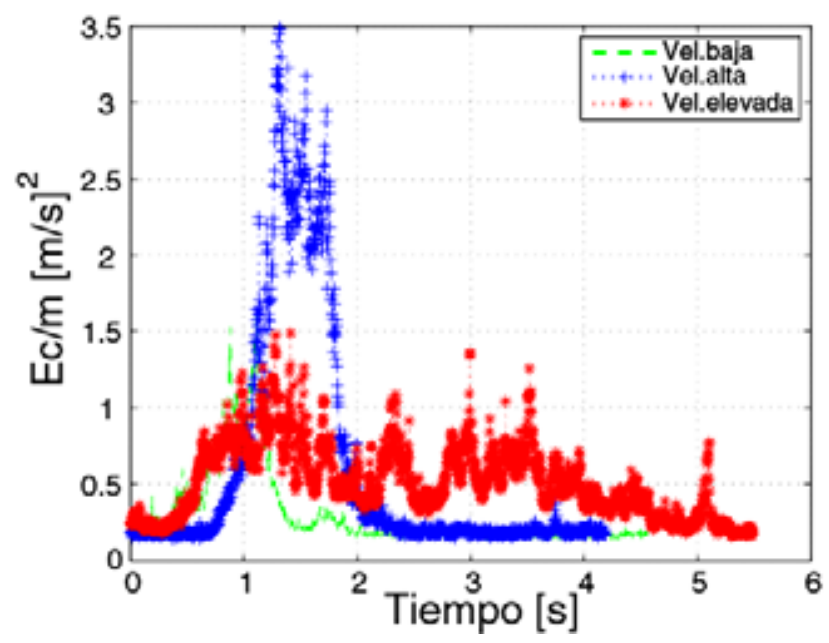

Figura 9. Variación de la energía cinética total versus tiempo para diferentes velocidades en canicas grandes.
9) con un comportamiento muy diferente a las canicas grandes. Se observa un claro desarrollo de la acumulación y disipación de energía en velocidad alta y en velocidad baja para la cual la energía es mucho menor; mientras en la velocidad elevada se presenta un proceso similar al observado con las canicas grandes, con una prolongación del evento de inestabilidad en diferentes picos de energía consecutivos y con un menor tiempo de flujo de material suelto.

En ensayos con arena de granulometría modificada (ver figura 10) se aprecia un patrón diferenciador en el valor máximo de la energía cinética. Este parte de un valor medio para una velocidad baja, incrementa para una velocidad alta y vuelve a valores mínimos de energía para una velocidad elevada, tal como se observa en la figura 10.

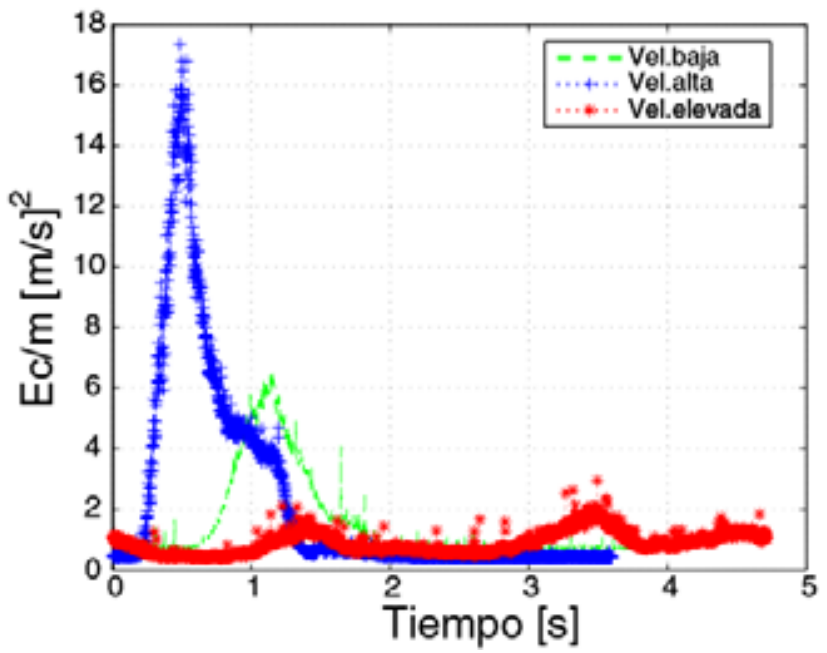

Figura 10. Variación de la energía cinética total con diferentes velocidades para la arena modificada.

En ambos materiales granulares se observa un comportamiento similar para las velocidades baja y alta, independientemente de la energía cinética alcanzada. Estas velocidades corresponden a un régimen de flujo en caída en donde se genera un incremento del ángulo de inclinación hasta alcanzar un valor máximo en el que se presenta el deslizamiento y disminuir hasta el ángulo de reposo (figura 11).

Por el contrario los materiales granulares en velocidades elevadas presentan un flujo de material constante (avalancha continua) y no es posible determinar un estado de reposo debido a la circulación de partículas entre la capa superior e inferior del talud. Esta velocidad corresponde a un régimen de flujo rodante (ver figura 12), tal como lo plantea Chou y Lee (2009). 

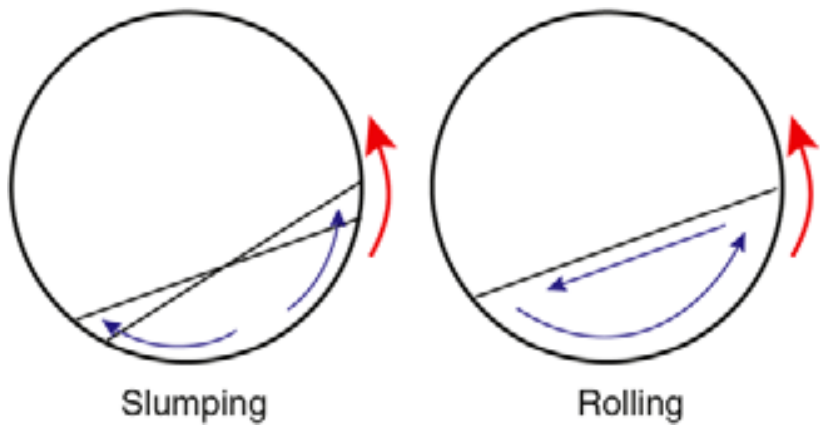

Figura 11. Interpretación de los regímenes de flujo registrados durante avalanchas de materiales granulares. Régimen de caída (slumping) y régimen rodante (rolling) Fuente: Chou and Lee, 2009.

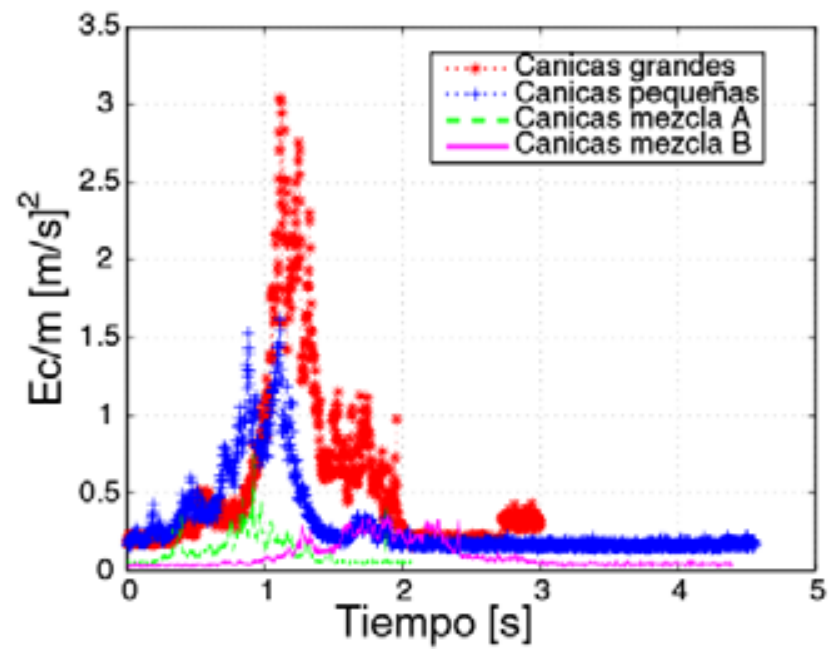

Figura 12. Variación de la energía cinética total versus tiempo para diferentes granulometrías en canicas con una velocidad de rotación alta.

\subsection{Granulometría}

$\mathrm{Al}$ analizar la influencia de la granulometría en los procesos de inestabilidad se puede observar que los materiales ensayados se comportan de modo similar.

En los materiales granulares artificiales como las canicas, los valores máximos de energía cinética existen en los materiales con un único tamaño de partícula. En las mezclas de canicas, el valor de la energía cinética disminuye debido a que la distribución espacial entre ellas no es uniforme como si lo es en las distribuciones de un solo tamaño.

De otro lado se puede apreciar que el tamaño de la partícula y su distribución en el tambor, influyen en la cantidad de energía que se pueda generar. En las canicas grandes pueden existir efectos de fricción o de efecto de arco en las partículas que generan una trabazón mayor entre ellas de tal forma que disipen una mayor cantidad de energía a diferencia de las canicas pequeñas.

Respecto a las arenas con granulometría modificada se observa que al existir una mejor distribución de partículas en la curva granulométrica, la organización de éstas dentro del tambor va a ser mejor de tal forma que el pico de energía disipado va a ser mayor. Con la granulometría original de las arenas no se alcanza a llegar a estos valores de energía cinética. En algunos casos se ve que se generan dos deslizamientos simultáneos, tal como se aprecia en la figura 13 en donde ocurre un deslizamiento inicial con bajos niveles de energía cinética y justo después se presenta el deslizamiento más grande que no alcanza a desarrollarse completamente durante el período de grabación del video de alta velocidad.

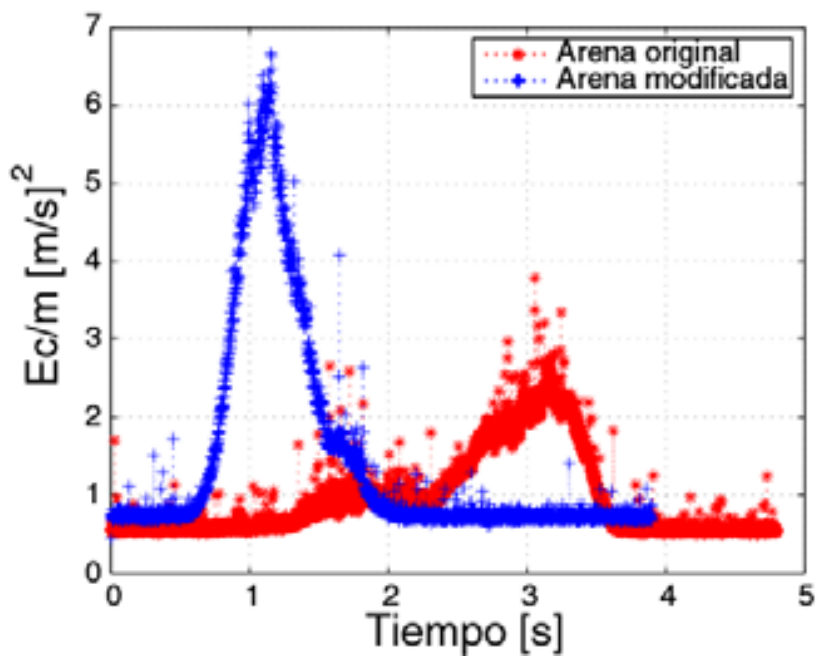

Figura 13. Variación de la energía cinética total para diferentes granulometrías de arena con velocidad de rotación baja.

\subsection{Probabilidad de ocurrencia}

La probabilidad de ocurrencia acumulada de un nivel de energía cinética, dentro del proceso de inestabilidad de los materiales a diferentes velocidades de rotación se describe en la figura 14.

Probabilidades del 25\% se interpretan como el nivel de la energía cinética de las partículas al inicio de la inestabilidad de los materiales. Las probabilidades del 50\% y 75\% determinan un nivel de energía cinética en 


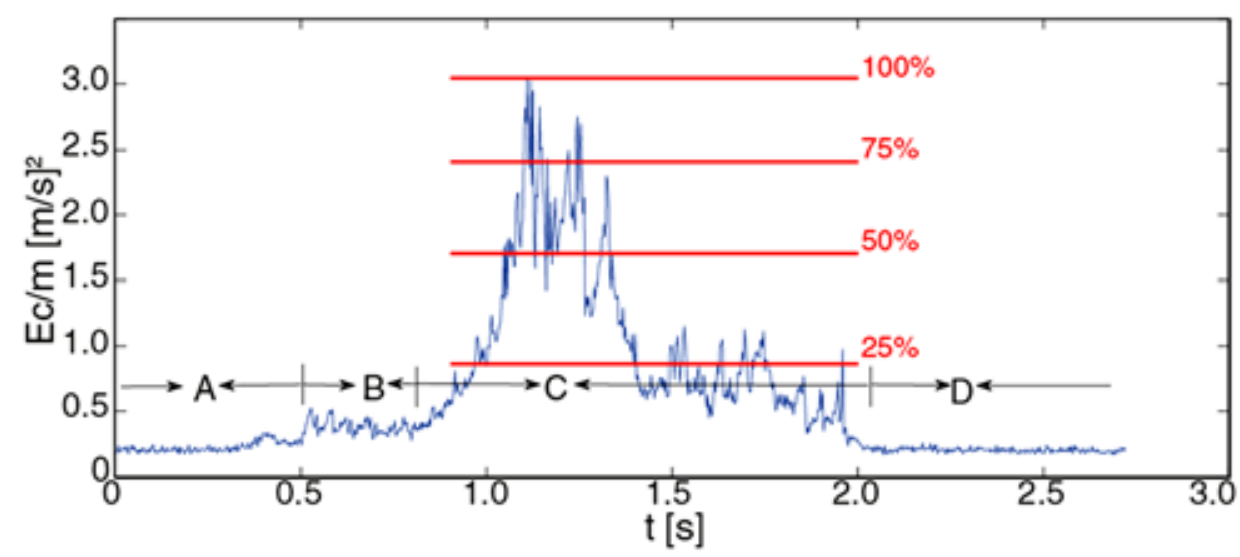

Figura 14. Probabilidades de ocurrencia de distintos niveles de energía cinética acumulada en el proceso de inestabilidad.

el que las partículas pierden completamente su estabilidad y comienza un proceso de flujo constante. Finalmente, las probabilidades del $100 \%$ representa la energía cinética máxima que alcanzan las partículas durante el proceso de inestabilidad.

La probabilidad de ocurrencia de un nivel de energía cinética tanto para canicas pequeñas (ver figura 15) como para arena modificada (ver figura 16) se analiza a la luz de la influencia de la velocidad de rotación del tambor.

Para las velocidades altas de rotación se presenta un comportamiento linealmente proporcional entre la probabilidad de ocurrencia y la energía cinética. Por el contrario, para velocidades bajas y elevadas de rotación, el comportamiento es proporcional pero no de tipo lineal, debido a que el rango de energía cinética alcanzada en el proceso de inestabilidad es menor.

De igual forma, se puede analizar la influencia de la granulometría de los materiales para una velocidad baja de rotación en las probabilidades de ocurrencia de niveles de energía cinética.

Se observa que para las canicas (ver figura 17) la probabilidad de ocurrencia describe un comportamiento más lineal y proporcional cuando las partículas son uniformes como lo son las canicas grandes y pequeñas y alcanza valores menores de energía cinética para las mezclas de tamaños.

Por el contrario en el caso de arenas, en la arena con granulometría modificada con mayor distribución de partículas se presenta un comportamiento más lineal y alcanza mayores valores de energía cinética que la arena de granulometría original.

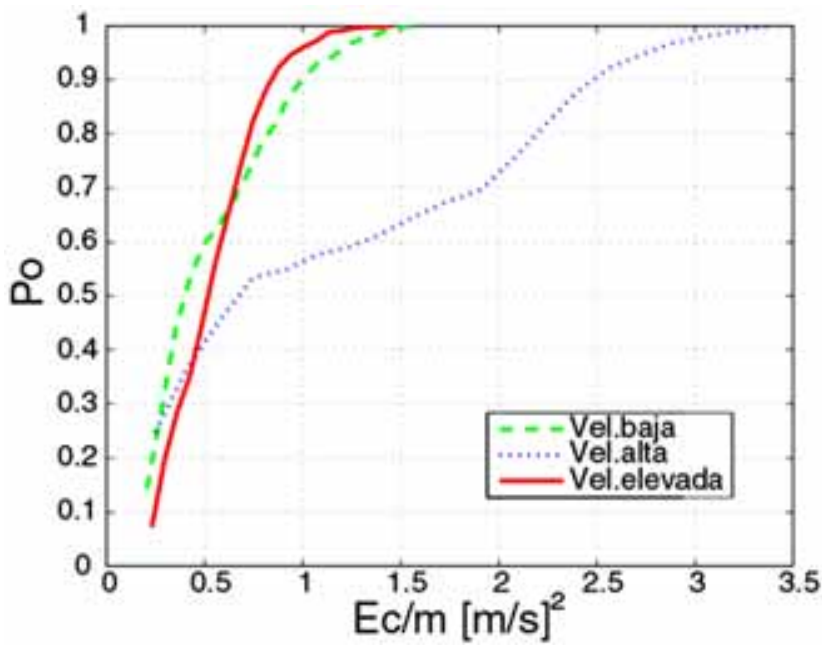

Figura 15. Variación de la probabilidad de ocurrencia de un nivel de energía cinética para diferentes velocidades de rotación en canicas pequeñas.

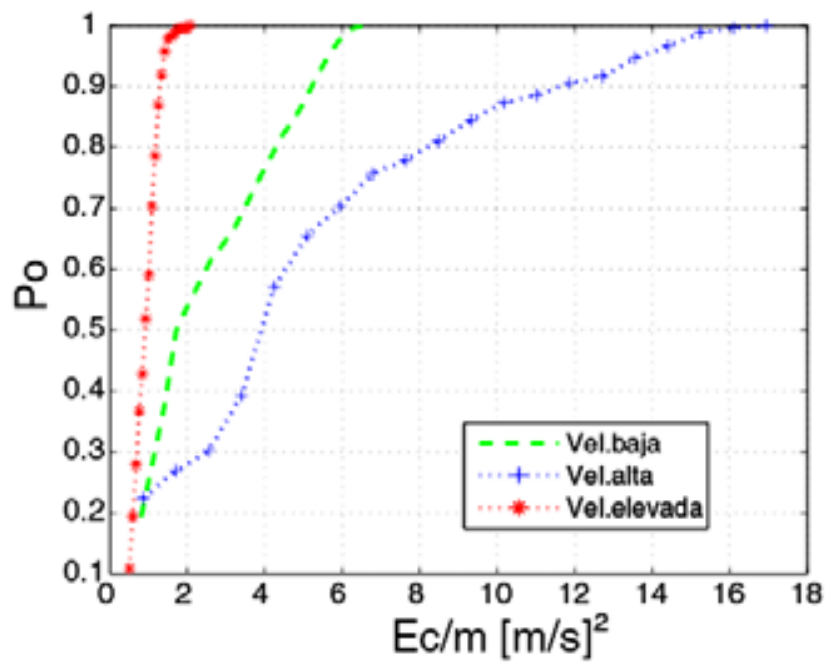

Figura 16. Variación de la probabilidad de ocurrencia de un nivel de energía cinética para diferentes velocidades de rotación de la arena modificada. 


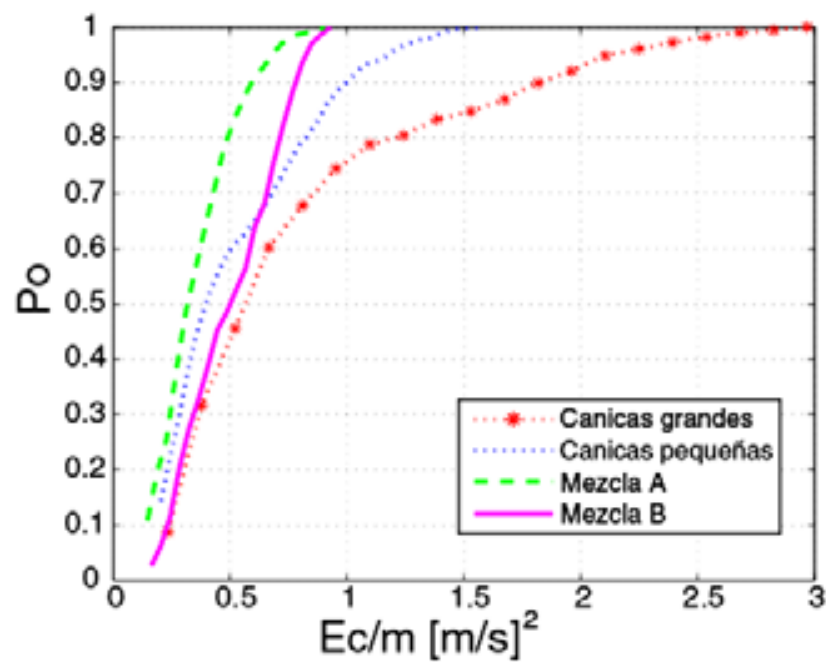

Figura 17. Variación de la probabilidad de ocurrencia de un nivel de energía cinética para diferentes granulometrías de canicas a una velocidad de rotación baja.

\section{Conclusiones}

- La velocidad de rotación del tambor determina dos regímenes de flujo en los materiales granulares. En el régimen de caída las partículas parten de un estado de reposo y se presenta un aumento súbito de la energía cinética en el inicio de la inestabilidad. Este proceso se observa cuando se aplican velocidades altas y bajas de rotación del tambor. En el régimen rodante se presenta un flujo constante de partículas representado por procesos de sucesivos de inestabilidad, con velocidades elevadas de rotación del tambor.

- La distribución de las partículas en el tambor influye en la magnitud de la energía que se puede disipar durante el proceso de inestabilidad. Con materiales de gradación uniforme como las canicas, se presentan valores picos mayores de energía cinética, posiblemente debido al efecto arco y a la homogeneidad de las partículas para canicas grandes y pequeñas, respectivamente. Por el contrario, una mejor gradación de las partículas en el ensayo de la arena con granulometría modificada produce valores de energía cinética más altos debido a la formación de un flujo constante de la avalancha.

- Este estudio muestra que la energía cinética puede ser empleada como un parámetro indicador del inicio del proceso de inestabilidad en materiales granulares al presentarse en un intervalo en el que existe un cambio significativo de pendiente y de inflexión en la curva de energía cinética en función del tiempo. Dicho nivel de energía cinética correspondería para las funciones empíricas de probabilidad de ocurrencia inicial de inestabilidades derivadas en este trabajo con un valor aproximado de la energía cinética normalizada de $0.5(\mathrm{~m} / \mathrm{seg})^{2}$ para las canicas y de $1.0(\mathrm{~m} / \mathrm{seg})^{2}$ para arenas, independientemente de la velocidad de rotación del tambor.

- La energía cinética de las partículas es un parámetro que brinda mejor información que los ángulos de inclinación de los materiales respecto al momento de inicio del proceso de inestabilidad. La variabilidad de la energía cinética inicial depende de la granulometría de los materiales. Dicho efecto de la granulometría del material no se observa en la fluctuación de los ángulos máximos de la superficie del material, ya que permanecen en un rango de $+3^{\circ}$ con respecto al ángulo de reposo, independientemente además de la velocidad de rotación del tambor.

\section{Referencias bibliográficas}

1. Bateman, A. a. M., 2007. Modelo bidimensional para la simulación de flujos detríticos: Flatmodel. Ingeniería Hidráulica de México, Volumen 22, pp. 5-20.

2. Centre for reearch for epidemiology of disasters, 2013. Natural disaster in 2012.

3. Chou, H. \& Lee, C., 2009. Cross sectional and axial flow characteristics of dry granular material in rotating drums. Granular Matter, pp. 13-32.

4. Jakob, M. \& Hungr, O., 2005. Debris- flow hazards and related phenomena. Springer.

5. Leonir, J. A., C.F.Ávila \& Hall, S., 2011. Multiscale modeling and characterization of ganular matter: From grain kinematics to continum mechanics. Journal of the Mechanics and Physics of Solids, Volumen 59, pp. 237-250.

6. Pust, O., 2000. Piv: Direct cross-correlation compared with fft-based cross-correlation. s.l., s.n.

7. Raffel, M., Willert, C. \& Kompenhans, J., 1998. Particle image velocimetry: a particle guide. s.l.:Springer. 
8. Slominski, C., Niedostatkiewicz, M. \& Tejchman, J., 2006. Deformation measurements in granular bodies using paticle image velocimetry. Archives of Hydro-Engineering and Environmental Mechanics, Volumen 53, pp. 71-94.

9. White, D., Take, W. \& Bolton, M., 2003. Soil deformation measurement using particle image velocimetry (PIV) and photogrammetry. Geotechnique, Volumen 53, pp. 619-631.
10.Xu, J. y otros, 2011. Quasi-real-time simulation of rotating drum using discrete element method with parallel GPU computing. Particuology, Volumen 9, pp. 446-450.

\section{Agradecimientos}

Los autores agradecen a la Pontificia Universidad Javeriana por el soporte económico de este trabajo con el proyecto No: 5267 "estudio experimental de la inestabilidad en un flujo de material granular seco". 